Binghamton University

The Open Repository @ Binghamton (The ORB)

2-18-2019

\title{
Dynamic Response of a Tunable MEMS Accelerometer Based on Repulsive Force
}

Meysam Daeichin

Binghamton University--SUNY, mdaeich1@binghamton.edu

Mehmet Ozdogan

Binghamton University--SUNY, mozdoga1@binghamton.edu

Shahrzad Towfighian

Binghamton University--SUNY, stowfigh@binghamton.edu

Ronald Miles

Binghamton University--SUNY, rmiles@binghamton.edu

Follow this and additional works at: https://orb.binghamton.edu/mechanical_fac

Part of the Mechanical Engineering Commons

\section{Recommended Citation}

Daeichin, Meysam; Ozdogan, Mehmet; Towfighian, Shahrzad; and Miles, Ronald, "Dynamic Response of a Tunable MEMS Accelerometer Based on Repulsive Force" (2019). Mechanical Engineering Faculty Scholarship. 23.

https://orb.binghamton.edu/mechanical_fac/23

This Article is brought to you for free and open access by the Mechanical Engineering at The Open Repository @ Binghamton (The ORB). It has been accepted for inclusion in Mechanical Engineering Faculty Scholarship by an authorized administrator of The Open Repository @ Binghamton (The ORB). For more information, please contact ORB@binghamton.edu. 


\title{
Dynamic Response of a Tunable MEMS Accelerometer Based on Repulsive Force
}

\author{
Meysam Daeichin, Mehmet Ozdogan, Shahrzad Towfighian ${ }^{* 1}$, Ronald Miles
}

\begin{abstract}
This paper describes a tunable MEMS electrostatic accelerometer that uses repulsive electrode configuration so that the design is not hampered by capacitive pull-in instability. The repulsive force configuration enables the increase of DC bias voltage without suffering from the pull-in failure mode. This flexibility in increasing voltage can be employed as a tuning parameter to widen the working frequency range and to improve the robustness of the accelerometer. A lumped parameter model is developed to simulate the response of the microstructure under a combination of electrostatic and dynamic mechanical loading. The electrostatic force is estimated using a finite element simulation. The nonlinear equations of motion are solved for harmonic base excitations and halfsine shock loads using the shooting and the long-time integration methods, respectively. To validate the model, a sensor is fabricated and characterized under harmonic base excitation and mechanical shocks. A mechanical sensitivity of $0.1 \frac{\mu \mathrm{m}}{g}$ is achieved when the bias voltage is $40(\mathrm{~V})$. The experimental data are in good agreement with the simulation results. The comprehensive dynamical characterization presented in this study contributes to the development of functional accelerometers with tunable capabilities to harmonic and shock accelerations.
\end{abstract}

(C) 2016

Keywords: MEMS sensor, MEMS Accelerometer, Repulsive Force, Pull-In Instability, Nonlinear Dynamics

\section{Introduction}

MEMS sensors and actuators have replaced their macro counterparts in many applications including, for example, pressure sensors, inertial navigation systems, and adaptive optic systems [1-3]. They have enabled consumer electronic products like smartphones, laptops, virtual reality headsets, and health monitoring wearable devices to deliver more enriched functionality. Their reliable performance, low cost, low power consumption, and more importantly, their compatibility with semiconductor fabrication technology have made MEMS technology popular.

Electrostatic actuation and detection is the most common transduction method in MEMS [4], and almost all the current commercially available electrostatic MEMS devices are based on parallel-plate [5] or interdigitated comb drive configurations [6]. In a parallel-plate configuration, the electrostatic force attracts a movable electrode to the substrate or bottom electrode. One can apply a specific voltage to move an electrode to a desired position in the case of an actuator (like micromirrors), or exert an excitation and sense change in the capacitance between two electrodes in the case of a sensor (like accelerometers). 
The main drawback of this electrostatic force is pull-in instability. Pull-in happens when the restoring force of the microstructure is no longer able to overcome the attractive electrostatic force and as a result, the microstructure collapses to its substrate. Capacitive MEMS transducers are designed to operate far from the pull-in region and this limits the device operation range; for example, it limits the travel range in micro-mirrors [7]. This issue is a significant constraint that needs to be considered in designing the microstructure geometry. Particularly, the initial gap between the movable electrode and the substrate is affected by this design limitation. This initial gap plays a crucial role in squeeze film damping [4] and the magnitude of the electrostatic force. As a result, it has a significant effect on the device sensitivity and performance in general.

As a new design paradigm, the repulsive capacitive sensing configuration is an alternative to parallel-plate or comb-drive configurations [8-10]. This approach takes advantage of the fringe electrostatic field to generate a repulsive force that pushes the movable structure away from the substrate, which essentially eliminates the possibility of pull-in (Fig. 1). This design approach opens a new door to build MEMS sensors and actuators without the limitation on performance imposed by dynamical instability from pull-in. Furthermore, because the movable electrode has the same voltage as the substrate (grounded), the possibility of micro-welding is eliminated. This failure mode happens when two microstructures with different voltages contact each other [11].

Lee et al. first introduced the repulsive approach [12]. A new generation of repulsive actuators was developed by He and Ben Mrad [8, 9, 13, 14]. The nonlinear dynamics of microstructures under a repulsive force has been investigated in the previous work of the authors [15-17]. In our previous work [15], we presented a comprehensive dynamical analysis of microbeams with clamped-clamped and cantilever boundary conditions under repulsive force. These microbeams are building blocks of this transduction scheme. We have investigated the nonlinear dynamic performance of a repulsive micro-mirror that showed significant travel ranges under parametric excitation [16]. However, to the best of our knowledge, there is no comprehensive study of the nonlinear dynamics of a sensing element in a repulsive electrode configuration.

In this study, we examine the repulsive approach to build a capacitive accelerometer. There are numerous applications for accelerometers. They are main components of inertial navigation systems used in cars, airplanes, smartphones, etc. For example, They are used to deploy airbags in automobiles in an accident [18] or, as a part of wearable health monitoring technologies, to detect the sudden fall of elderly people[19] to provide them with necessary medical attention as soon as possible. For a comprehensive study on accelerometer sensors based on the parallel-plate scheme, see [20].

The electrostatic force on the moving element in the system examined here differs considerably from that found in conventional parallel-plate electrode designs and cannot be estimated using simple analytical expressions. This, along with the large amplitude vibration of the proof-mass, make the dynamics of the system fully nonlinear and complicated. Therefore, our present focus is on exploring the dynamics of the sensing element as it vibrates in electric field. This should be accomplished prior to the development of a read-out circuit, which will be addressed in a subsequent work.

The structure of this paper is organized as follows. In Section 2, the working principle of the sensor design is explained. In Section 3, a mathematical model of the structure is developed, and a lumped parameter model is extracted. Section 4 presents the fabrication process of the device. 
Experimental results from dynamic tests on the fabricated device are presented and discussed in Section 5 followed by a conclusion.

\section{Structure design and working principle}

The general schematic of the structure under base excitation is shown in Fig.1. There are four elements that comprise this design. The first element is the square plate that is attached to its anchors through two torsional beams. The two beams undergo torsional deflection as the proof mass rotates because of applied acceleration and electrostatic forces. The second part is the $200 \mu \mathrm{m}$ long micro-beams that are attached to three sides of the proof mass. The proof-mass and fingers attached to it are all electrically grounded. Under these fingers, there are sets of grounded fingers fixed on the substrate as the third element of the sensor. The fourth part is the sets of electrodes adjacent to the grounded fingers. These fingers carry electrical voltage, the electric field of which exerts electrostatic forces on the beams connected to the proof mass.

The dimensions of all these beams and their electrostatic field are illustrated in Fig. 1. The fringe electrostatic field exerts different forces on the top and bottom surfaces of the moving fingers. The resultant force is an upward force that pushes the fingers and the proof mass away from the substrate. Beyond a certain distance, the electrostatic force becomes attractive and tends to bring the microstructure back into the repulsive region. Therefore, there is an equilibrium point where the electrostatic force on the structure becomes zero. Once a voltage is applied on the side electrodes, the accelerometer plate rotates away from the substrate to an equilibrium position where the electrostatic force is equal to the restoring force of the torsional springs. This equilibrium point can be tuned by changing the voltage on the side electrodes. As will be discussed below, this is advantageous when we want to set the threshold acceleration that the sensor can detect. When the base of the plate accelerates, the plate tends to stay at rest because of its inertia and this leads to a relative displacement between the base and the plate. Because of this displacement, the capacitance between the electrodes will change as well. This change in capacitance can be measured and related to the relative motion of the plate. If the load is large enough, the fingers can hit the bottom electrodes. However, because of the repulsive nature of the force, they will bounce back and will remain free to move. This collision can be detected with the aid of an electrical circuit. Our main focus here is to investigate the motion of the microstructure under the combined nonlinear electrical and mechanical loads as understanding this behavior is an essential part of the sensor characterization for making functional MEMS accelerometers. In the next section, the mathematical model of the structure will be presented to explore the dynamical characteristics of the system.

\section{Mathematical modeling}

The mathematical model presented in this section can be used as a tool to refine the sensor design before going through the fabrication process. As shown in our previous work [16], this simple single degree of freedom model is a very powerful tool to predict the response of the system and capture the inherent nonlinearity caused by the electrostatic force. We consider a 

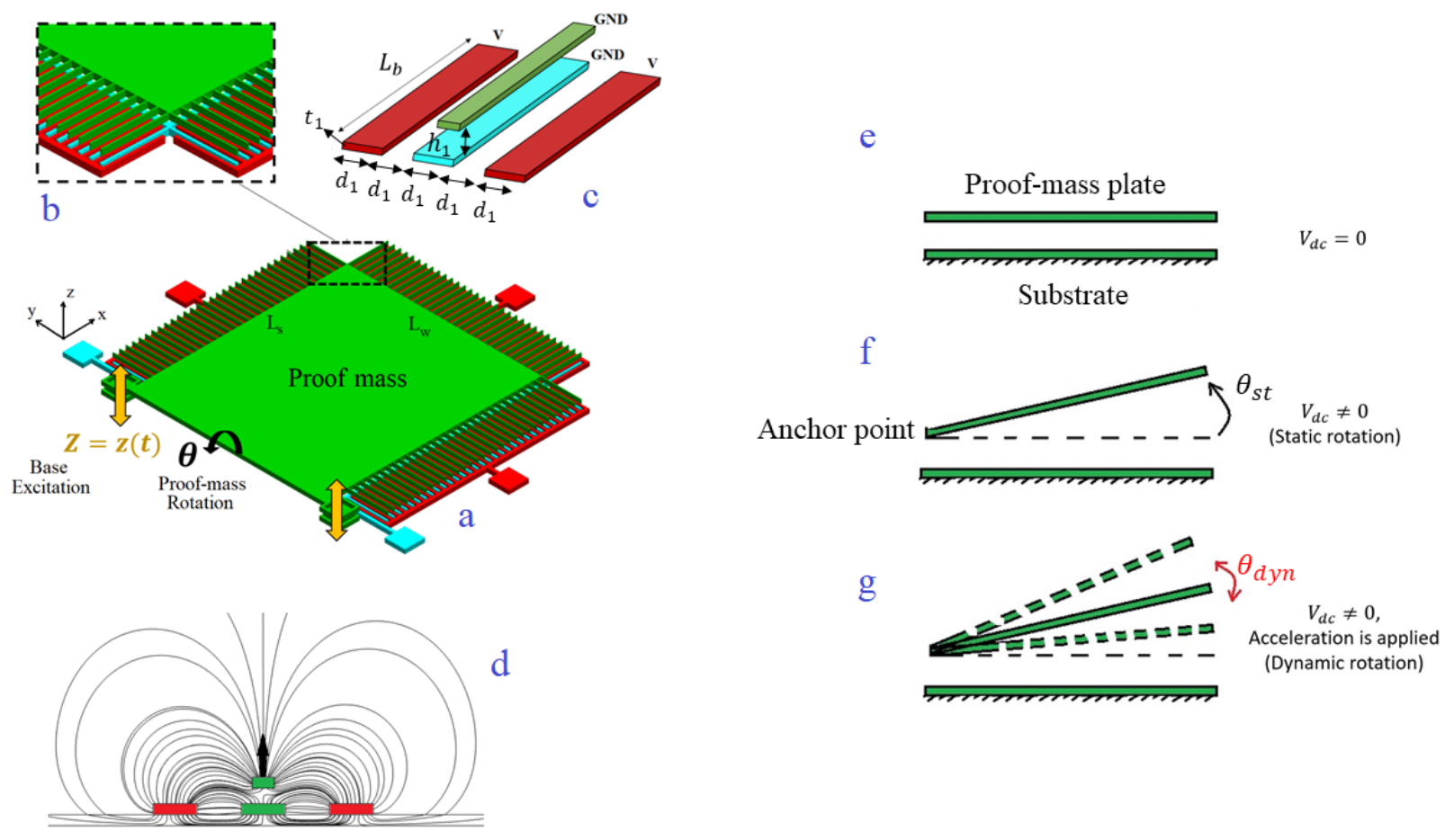

Figure 1: (a) The general schematic of the accelerometer. Base excitation (shown by yellow bidirectional arrows) will be transferred to the microstructure through its supports causing the plate to rotate. (b) A closer view of the electrodes configuration. (c) Voltage distribution on beams. (d) Asymmetric fringing electric field exerts an upward force on the moving electrode (e) Side view of accelerometer and substrate when $V_{d c}=0(\mathbf{f})$ when $V_{d c} \neq 0$ but there is no mechanical load and (g) When $V_{d c} \neq 0$ and a mechanical load is applied.

lumped parameter oscillator as the following:

$$
I \hat{\ddot{\theta}}+c \hat{\dot{\theta}}+k \hat{\theta}=M_{e s}(\hat{\theta})+M_{s h}
$$

in which $\hat{\theta}$ is the rotation of the proof-mass plate about its anchors' axis, $I, c$ and $k$ are the moment of inertia, torsional damping, and torsional stiffness coefficients, respectively. On the right-hand side, $M_{e s}$ and $M_{s h}$ represent the nonlinear moments caused by the electrostatic and base acceleration forces, respectively.

The electrostatic force has a nonlinear dependence on the position of the moving fingers in the electric field. To obtain an expression for the electrostatic moment, first we calculate the force profile for a unit-cell using a 2D finite element analysis in COMSOL. Such a unit cell consists of the moving electrode, bottom electrode and two side electrodes as shown in Fig. 1. The force profile is shown in Fig. 2.

Using a curve fitting technique, the force profile (Fig. 2) can be approximated as a $9^{\text {th }}$-degree 


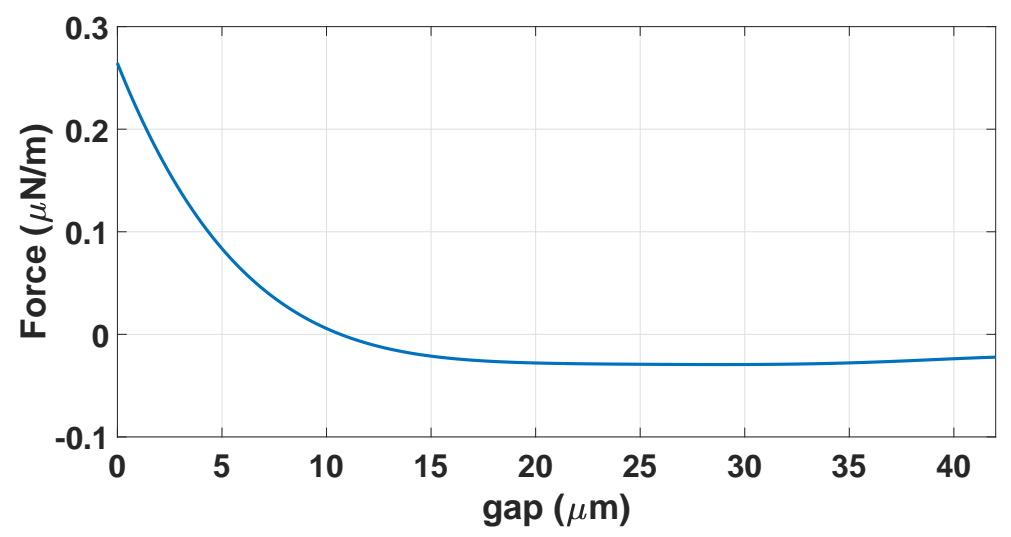

Figure 2: Electrostatic force profile obtained from COMSOL for the two-dimensional unit cell of the sensor

polynomial, in terms of the deflection, $z$.

$$
F_{e s}=V_{d c}^{2} \sum_{i=0}^{9}\left(A_{i} z^{i}\right)
$$

where $z$ is the gap between the bottom electrode and moving fingers in each cross section. In order to write the electrostatic force as a function of $\theta$, we use the following trigonometric equation.

$$
\sin \theta=\frac{z}{x}
$$

which yields

$$
F_{\text {es-mov-fing }}(x, \theta)=V_{d c}^{2} \sum_{i=0}^{9} A_{i}(x \cdot \sin \theta)^{i}
$$

Integrating Eq. (4) over the finger length results in the total electrostatic force on each beam.

$$
\begin{array}{r}
F_{\text {es-tip }}(\theta)=\int_{L_{s}}^{L_{s}+L_{b}} F_{\text {es-mov-fing }}(x, \theta) d x= \\
\int_{L_{s}}^{L_{s}+L_{b}} V_{d c}^{2}\left(\sum_{i=0}^{9} A_{i}(x \cdot \sin \theta)^{i}\right) d x \\
=V_{d c}^{2} \sum_{i=0}^{9} \frac{A_{i}(\sin \theta)^{i}}{i+1} \cdot\left(\left(L_{s}+L_{b}\right)^{i+1}-L_{s}^{i+1}\right)
\end{array}
$$

Where the parameters are given in Table 1. To calculate the corresponding moment caused by this force, the distance between the acting point of the resultant force and the axis of rotation $\left(x_{c}\right)$ is needed, which can be calculated as follows:

$$
x_{c}=\frac{\int_{L_{s}}^{L_{s}+L_{b}} x \cdot F_{\text {es-mov-fing }} d x}{\int_{L_{s}}^{L_{s}+L_{b}} F_{\text {es-mov-fing }} d x}
$$


Therefore, the moment of electrostatic force on the tip fingers about the axis of rotation can be written as

$$
\begin{array}{r}
M_{e s-t i p}=N_{t} \cdot F_{e s-t i p}(\theta) \cdot x_{c}= \\
N_{t} V_{d c}^{2} \sum_{i=0}^{9} \frac{A_{i}(\sin \theta)^{i}}{i+2} \cdot\left(\left(L_{s}+L_{b}\right)^{i+2}-L_{s}^{i+2}\right)
\end{array}
$$

where $N_{t}$ is the number of fingers on the tip side of the proof mass. Following a similar approach and assuming that the electrostatic force on the fingers on the sides of the proof-mass does not change over the finger length, we can calculate the moment of the electrostatic force on these fingers as follows.

$$
M_{e s-s i d e}=V_{d c}^{2} \cdot \sum_{j=0}^{N s} \sum_{i=0}^{9}\left(A_{i}\left(u_{j} \cdot \sin \theta\right)^{i} \cdot L_{b}\right) \cdot u_{j}
$$

Where $u_{j}$ is the distance of the $j^{\text {th }}$ side finger from the axis of rotation and $N_{s}$ is the number of fingers on each side. So, the total electrostatic moment in Eq. (1) can be written as:

$$
M_{e s}(\hat{\theta})=M_{e s-t i p}+2 \cdot M_{e s-s i d e}
$$

The factor 2 in Eq. (9) accounts for the fact that there are two sets of side fingers, one on each side of the proof-mass. The moment from acceleration $\left(M_{s h}\right)$ also can be written as:

$$
\begin{array}{r}
M_{s h}=\left[m_{p} \cdot a(t) \cdot \frac{L_{s}}{2}\right]+\left[N_{t} \cdot m_{b} \cdot a(t) \cdot\left(L_{s}+\frac{L_{b}}{2}\right)\right]+ \\
{\left[2 \cdot\left(\sum_{j=1}^{N_{s}} m_{b} \cdot a(t) \cdot u_{j}\right)\right]}
\end{array}
$$

where the first bracket is the moment caused by proof mass acceleration, the second shows the moment caused by the tip fingers acceleration, and the third is for the side fingers. In Eq. (10), $m_{p}$, $m_{b}$, and $a(t)$ are the mass of the rotational plate, mass of the moving electrode, and the acceleration load that is exerted on the microstructure through base excitation. This mechanical load can be modeled as a harmonic base excitation or as a shock load. There are different shock profiles commonly used for modeling shock loads such as square, saw-tooth, half-sine or full-sine shock profiles. The most commonly used shock load is the half-sine with the profile given in Eq. (11), which will be used in the following in our description of the experiment on the fabricated device,

$$
a(t)=A \sin (\omega t) \cdot u\left(\frac{\pi}{\omega}-t\right)
$$

Where $u\left(\frac{\pi}{\omega}-t\right)$ is the step function. Dividing Eq. (1) by $I$ and using Eq. (9) and Eq. (10) and following non-dimensional parameters (Eq. (12)), we can rewrite the governing equation of motion in non-dimensional format (Eq. (13)).

$$
\theta=\frac{\hat{\theta}}{\theta_{0}}, \quad t=\frac{\hat{t}}{T}, \quad T=\frac{1}{\omega_{n}}, \quad \omega_{n}=\sqrt{\frac{k}{I}}
$$




$$
\ddot{\theta}+2 \xi \omega_{n} \dot{\theta}+\omega_{n}^{2} \theta=\frac{T^{2}}{I \theta_{0}} \cdot\left(M_{e s}(\theta)+M_{s h}\right)
$$

As discussed in Section 2, by applying a DC voltage on the side electrodes, the proof-mass rotates around its anchors' axis and goes to an equilibrium point away from the substrate. To obtain this static rotation, all the time derivative terms in Eq. (13) are set to zero and then the equation is solved for static rotation $\left(\theta_{s t}\right)$. The static rotation can be used to solve for the dynamic solution $\left(\theta_{d y n}\right)$ of Eq. (13) in the presence of any time-varying load using the following change of variable.

$$
\theta=\theta_{s t}+\theta_{d y n}
$$

The moment of inertia, $I$, in Eq. (13) can be calculated as

$$
\begin{array}{r}
I=\frac{1}{3} m_{p} L_{s}^{2}+N t \cdot\left(\frac{1}{12} m_{b} L_{b}^{2}+m_{b}\left(\frac{L_{b}}{2}+L_{s}\right)^{2}\right)+ \\
\sum_{j=1}^{N_{s}}\left(\frac{1}{12} m_{b} b^{2}+m_{b} u_{j}^{2}\right)
\end{array}
$$

The natural frequency of the first mode, $\omega_{n}$ is obtained from the ANSYS finite element package. The first mode is a rotational mode as expected. The corresponding natural frequency for this mode is predicted to be $1320(\mathrm{~Hz})$. After examining our subsequent experimental data, we can modify this value for the natural frequency to account for all the fabrication imperfections, residual stresses and mathematical simplification in this 1 DOF model.

Equation (13) can be solved numerically using the Runge-Kutta method for various damping conditions. However, it is likely that a simple linear damping does not capture the physics of energy dissipation in this problem. In general, modeling energy dissipation in the repulsive scheme is more challenging compared to the attractive scheme because of two reasons. First, the amplitude of vibration can get very large compared to the initial fabrication gap because the initial static deflection of the structure (from $V_{d c}$ ) moves the structure away from the substrate, providing more space for vibration. Therefore, those damping models available in the literature [21-23] that are based on Reynolds' equation are not valid because the underlying assumptions such as negligible pressure change across the fluid film or small gap to lateral dimension ratio are not valid here. Second, because there is no limitation from pull-in, the device can get very close to the substrate while having a large amplitude vibration. So, in each cycle of vibration, when the moving part is far away from the substrate the dominant damping mechanism is the drag force in free air. However, when the structure gets close to the substrate, the squeeze film damping mechanism starts to play a significant role in energy dissipation. Thus, the damping force is likely to be quite nonlinear.

Another challenge in modeling damping in this problem is that the continuity assumption for the air breaks down when the microstructure gets very close to the substrate, especially at low pressures. In this situation, the characteristic length of the problem is even smaller than the initial fabrication gap, and the characteristic length of the problem becomes comparable with the mean 
free path of air molecules. The Knudson number is the parameter to check to see if the continuity assumption is valid (Eq. (16)). This number is defined as the ratio of the mean free path of air (gas) molecules to the characteristic length of the problem, which is usually the gap between moving and stationary parts of the microstructure. The mean free path of air molecules depends on the air temperature and pressure. Assuming the room temperature condition $\left(25^{\circ} \mathrm{C}\right)$, the dependence of the molecules' mean free path on pressure can be written as in Eq. (16).

$$
K_{n}=\frac{\lambda}{H}, \quad \lambda=\frac{P_{0}}{P} \lambda_{0}
$$

Using the Knudsen number, the flow is divided into four regimes: continuum flow, slip flow, transitional flow and free molecular flow. Assuming the characteristic length to be equal to the 


\begin{tabular}{|c|c|c|c|}
\hline Parameter & Symbol & Value & Unit \\
\hline Density & $\rho$ & 2330 & $\frac{\mathrm{kg}}{\mathrm{m}^{3}}$ \\
\hline Proof mass length & $L_{s}$ & 990 & $\mu \mathrm{m}$ \\
\hline Proof mass width & $L_{w}$ & 1000 & $\mu \mathrm{m}$ \\
\hline finger length & $L_{b}$ & 200 & $\mu \mathrm{m}$ \\
\hline number tip fingers & $N_{t}$ & 31 & $\mu \mathrm{m}$ \\
\hline number side fingers & $N_{s}$ & 25 & $\mu \mathrm{m}$ \\
\hline Voltage fixed finger width & $d_{1}$ & 8 & $\mu \mathrm{m}$ \\
\hline Gap between fixed fingers & $d_{1}$ & 8 & $\mu \mathrm{m}$ \\
\hline Moving finger width & $d_{2}$ & 4 & $\mu \mathrm{m}$ \\
\hline Ground fixed finger width & $d_{2}$ & 8 & $\mu \mathrm{m}$ \\
\hline Electrodes and Proof thickness & $t_{1}$ & 2 & $\mu \mathrm{m}$ \\
\hline Initial gap & $h_{1}$ & 2 & $\mu \mathrm{m}$ \\
\hline Natural frequency & $\omega_{n}$ & $2 \times \pi \times 1740$ & $\frac{\mathrm{rad}}{\mathrm{s}}$ \\
\hline Damping ratio $(V d c=40)$ & $\xi_{0}$ & 0.016 & \\
\hline Damping ratio $(V d c=40)$ & $\xi_{1}$ & 0.033 & \\
\hline Damping ratio $(V d c=50)$ & $\xi_{0}$ & 0.016 & \\
\hline Damping ratio $(V d c=50)$ & $\xi_{1}$ & 0.038 & \\
\hline Damping ratio $(V d c=60)$ & $\xi_{0}$ & 0.017 & \\
\hline Damping ratio $(V d c=60)$ & $\xi_{1}$ & 0.055 & \\
\hline Force Constant & $A_{0}$ & $3.4079 \times 10^{-7}$ & $N / m$ \\
\hline Force Constant & $A_{1}$ & $-6.7113 \times 10^{-2}$ & $N / m^{2}$ \\
\hline Force Constant & $A_{2}$ & $7.5644 \times 10^{3}$ & $N / m^{3}$ \\
\hline Force Constant & $A_{3}$ & $-8.8555 \times 10^{8}$ & $N / m^{4}$ \\
\hline Force Constant & $A_{4}$ & $8.1341 \times 10^{13}$ & $N / m^{5}$ \\
\hline Force Constant & $A_{5}$ & $-4.6766 \times 10^{18}$ & $N / m^{6}$ \\
\hline Force Constant & $A_{6}$ & $1.6139 \times 10^{23}$ & $N / m^{7}$ \\
\hline Force Constant & $A_{7}$ & $-3.2602 \times 10^{27}$ & $N / m^{8}$ \\
\hline Force Constant & $A_{8}$ & $3.5560 \times 10^{31}$ & $N / m^{9}$ \\
\hline Force Constant & $A_{9}$ & $-1.6175 \times 10^{35}$ & $N / m^{10}$ \\
\hline Moment of inertia & $I$ & $1.7954 \times 10^{-15}$ & $k g \times m^{2}$ \\
\hline
\end{tabular}

Table 1: Dimensions for the MEMS accelerometer in Figure 1.

\begin{tabular}{|c|c|}
\hline Pressure (Torr) & regime \\
\hline$P>2200$ & Continuum \\
$1000<P<2200$ & Slip flow \\
$10<P<1000$ & Transient \\
$P<10$ & Molecular \\
\hline
\end{tabular}

Table 2: Air flow regimes for characteristic length $H=2 \mu m$

initial gap and using equations (16) we can categorize the flow according to pressure (Table 
2). As we will be performing the experiment on the device at very low pressure $(P<350 m$ Torr $)$, the flow regime would be in the free molecular regime. Also, as the microstructure gets very close to the substrate, the characteristic length of the problem becomes even smaller than $2 \mu m$, which leads to higher threshold pressure for the molecular region. In our previous work, we proposed a modular damping that captured the complicated physics of this energy dissipation mechanism. The proposed modular damping depends on the ratio of vibration amplitude to the initial gap (initial fabrication gap + static deflection caused by DC voltage). When the vibration amplitude is small compared to the initial gap, the damping is dominated by linear viscous damping. However, as the vibration amplitude becomes comparable with the initial gap, the squeeze film damping increases as the air molecules are trapped between the proof mass and substrate. The equation accounting for this variable damping that modulates itself with the amplitude-gap ratio is given in Eq. (17) [16].

$$
\xi=\xi_{0}+\xi_{1}\left(\frac{\left|\theta_{\text {dyn }}\right|}{\theta_{\text {initial }}}\right)
$$

With the description of damping above, all the parameters in Eq. (13) have been defined. Next, the fabrication process is explained followed by experimental results.

\section{Fabrication Process}

The process flow of the device fabrication is depicted in Figure 3. It starts with 4-inch standard silicon wafers which are dipped into base and acid baths (RCA Cleaning). This process removes residues such as organic particles and the native oxide on the wafer surface. After the cleaning, we grew $1 \mu m$ thick oxide as an insulation layer using a low-pressure chemical vapor deposition (LPCVD) furnace. On top of this layer, we deposited $0.2 \mu \mathrm{m}$ thick low-stress nitride. The oxide and nitride layers serve as insulators that isolate the device from the substrate. On top of the nitride layer, a $2 \mu \mathrm{m}$ thick phosphorus-doped amorphous silicon (N+ a-Si) was deposited in a LPCVD furnace. The deposition rates and the deposition temperatures of the LPCVD films are presented in Table 3. This amorphous layer was then formed to polycrystalline silicon (poly-Si) by an annealing process $[24,25]$. Following the annealing, we spun a $2 \mu \mathrm{m}$ thick positive photoresist and exposed it with an i-line stepper. Exposed wafers were baked at $115{ }^{\circ} \mathrm{C}$ for 90 seconds and developed with 726 MIF developer for 60 seconds. After the developing step, the first layer of fingers was created via the Bosch etching process. The chamber conditions of the etching process are presented in Table 4. On top of the fixed fingers, we deposited $4 \mu \mathrm{m}$ thick high-temperature oxide (HTO) film as a sacrificial layer. This layer provides a vertical gap between the first and second poly-Si films which are the fixed and the moving fingers, respectively. Following the deposition process, we planarized $2 \mu \mathrm{m}$ of the oxide using a Chemical Mechanical Polisher (CMP). This process removes the step difference between the proof mass and the fixed fingers. Later, using Inductively Coupled Plasma Reactive Ion Etching (ICP-RIE), we etched the sacrificial oxide to open vias between first and second poly-Si. The chamber condition of this process is presented in Table 4. Upon etching the vias, we deposited another layer of $2 \mu \mathrm{m}$ thick phosphorus-doped a-Si which is also annealed to form poly-Si as done before. This layer is etched to form the moving fingers that are attached to the rectangular shaped proof mass. After the etching, $20 \mathrm{~nm} \mathrm{Cr}$ and $150 \mathrm{~nm}$ of Au are deposited 


\begin{tabular}{cccc}
\hline Film & Temp. $\left({ }^{\circ} \mathrm{C}\right)$ & Thickness $(\mu m)$ & Dep. Rate $(\AA / m i n)$ \\
\hline & & & \\
$\mathrm{SiO}_{2}$ & 1100 & 1 & 74 \\
$\mathrm{Si}_{3} \mathrm{~N}_{4}$ & 800 & 0.2 & 25 \\
$\mathrm{HTO}$ & 800 & 4 & 12.7 \\
$\mathrm{a}-\mathrm{Si}$ & 570 & 2 & 11.7 \\
Annealing & 1000 & - & - \\
& & & \\
\hline
\end{tabular}

Table 3: Information related to the thin film depositions.

Bosch Process for Silicon Etching

\begin{tabular}{cccccccc}
\hline Process & Pressure (mTorr) & $\mathrm{C}_{4} \mathrm{~F}_{8}(\mathrm{sccm})$ & $\mathrm{SF}_{6}(\mathrm{sccm})$ & $\mathrm{Ar}(\mathrm{sccm})$ & $\mathrm{RF} 1$ & $\mathrm{RF} 2$ & Duration (sec) \\
\hline & & & & & & & \\
Etch 1 & 23 & 2 & 100 & 40 & 8 & 850 & 5 \\
Passivation & 24 & 70 & 2 & 100 & 0.1 & 850 & 5 \\
Etch 2 & 23 & 2 & 70 & 40 & 8 & 850 & 2 \\
Etch 3 & 23 & 2 & 100 & 40 & 8 & 850 & 5 \\
\hline
\end{tabular}

ICP Process for Oxide Etching

\begin{tabular}{cccccccc}
\hline Process & Pressure (mTorr) & $\mathrm{CHF}_{3}(\mathrm{sccm})$ & $\mathrm{O}_{2}(\mathrm{sccm})$ & $\mathrm{Ar}(\mathrm{sccm})$ & $\mathrm{RIE}(\mathrm{W})$ & $\mathrm{ICP}(\mathrm{W})$ & Duration $(\mathrm{sec})$ \\
\hline Etch & 5 & 52 & 2 & - & 15 & 2500 & - \\
\hline
\end{tabular}

Table 4: Chamber conditions for dry etching. Etch rates for silicon and oxide are measured around $2 \mu \mathrm{m} / \mathrm{min}$ and 150 $\mathrm{nm} / \mathrm{min}$, respectively.

on the pads using an e-beam evaporation tool. Then the wafers are diced and released in $\mathrm{HF}: \mathrm{HCl}$ solution. Following the release, the chips are critical point dried (CPD) to avoid stiction. The scanning electron microscope (SEM) images of the fabricated device are presented in Figure 4. The chip is mounted on a printed circuit board and wire-bonded using an aluminum wire wedgebonder.

\section{Experimental Setup and Results}

We conducted experiments on the fabricated sensor to investigate the accelerometer dynamical performance and verify the mathematical model that was developed in section 3 . The sensor was subjected to two different mechanical loads, harmonic excitations and shock loads. For harmonic loading, the dynamic response of the microstructure in the resonance region will be discussed that sheds light on the effects of inherent nonlinearities in the electrostatic force. Also, the mechanical sensitivity of the accelerometer will be obtained which is an important parameter with a significant contribution to the overall sensitivity of the sensor. This mechanical sensitivity is obtained from 

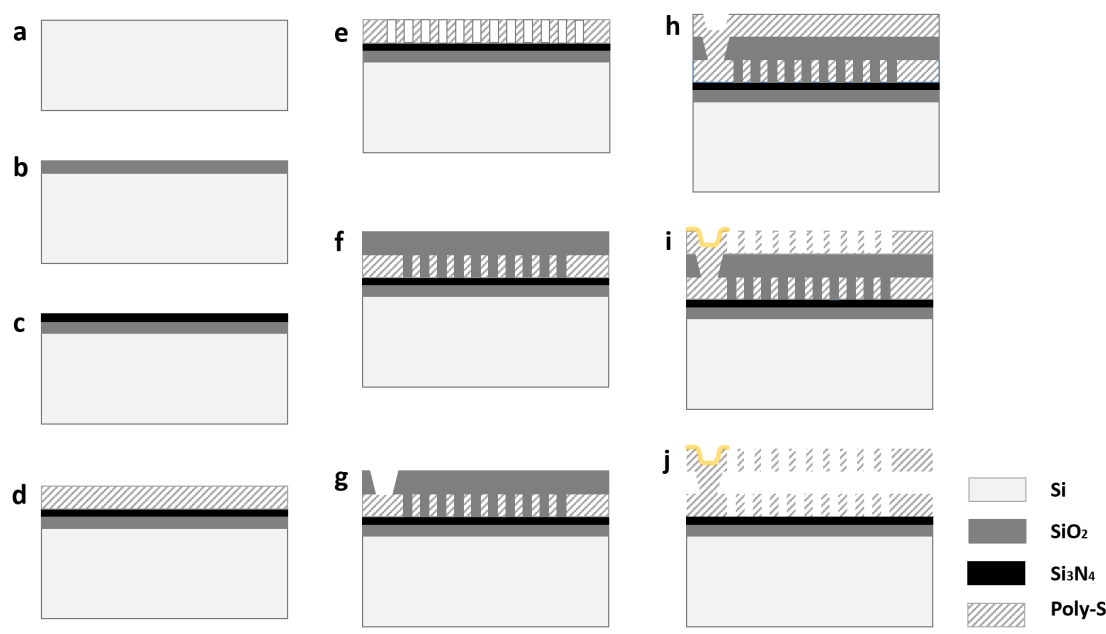

Figure 3: Fabrication process (a) 4-inch, $525 \mu \mathrm{m}$ thick bare Si wafer. (b-c) Insulation layers growth and deposition. (d) First polysilicon deposition. (e RIE etch of polysilicon. (f) Sacrificial layer deposition and CMP processing. (g) Anchor etch on a sacrificial layer. (h) Second polysilicon deposition. (i) Polysilicon etch and gold deposition on the pads. (j) Release in $\mathrm{HF}: \mathrm{HCl}$ solution and CPD.
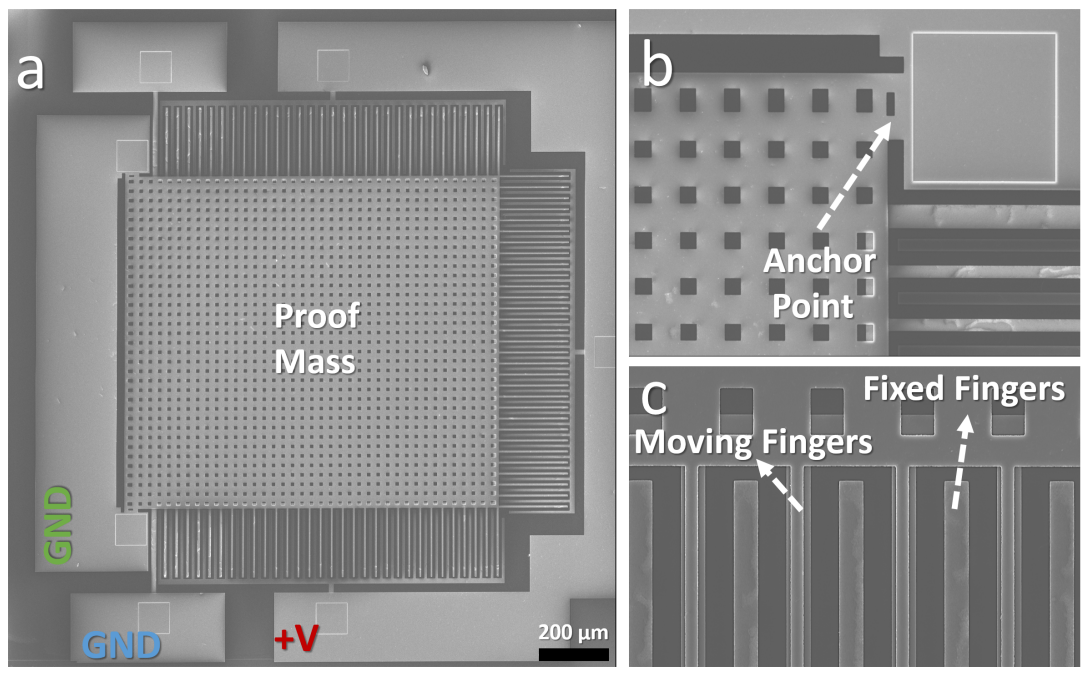

Figure 4: (a) SEM image of the device from top. (b) Closer view of the device anchor point. (c) Moving and fixed electrode configuration.

the frequency response before reaching the resonance region because accelerometers are always used at frequencies below their resonance range. The robustness of the accelerometer against mechanical shocks is another important parameter that will be analyzed in the following.

The printed circuit board containing the MEMS chip is mounted to the head of the shaker and placed inside the vacuum chamber at 200mTorr. A laser vibrometer (Polytec MSA-500) is used 
to monitor the time-response of the sensor. This set up is shown in Fig. 5. The next part addresses how the shaker acceleration is kept constant while sweeping the frequency.

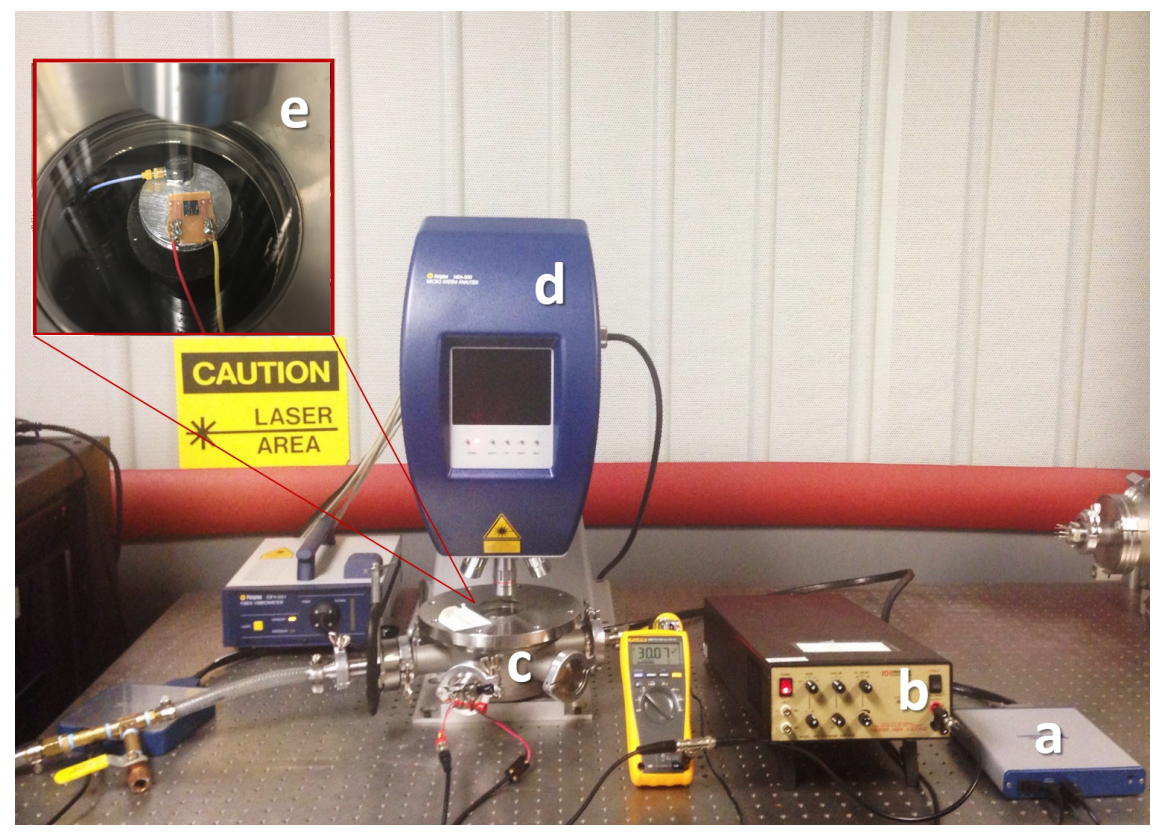

Figure 5: Experimental setup. (a) NI-USB 6366 Data Acquisition Box (b) Krohn-Hite 7600 power amplifier. (c) Vacuum chamber. (d) MSA-500 Polytec Laser Doppler Vibrometer. (e) Shaker inside the vacuum chamber and the chip attached on the PCB.

\subsection{Shaker Characterization}

The output acceleration of the shaker depends on the amplitude and frequency of the voltage signal given to the shaker. To conduct a frequency sweep on the microstructure, the shaker needs to provide a base acceleration with a constant amplitude at different frequencies. Usually, a closed loop system is used with the shaker where the output acceleration of the shaker is constantly monitored so acceleration amplitude can be kept constant by modifying the amplitude of the voltage signal. Here, the shaker is used in an open loop mode. To identify the required voltage needed to send to the shaker at each frequency $(f)$, the shaker is characterized in a separate setup where we use the laser vibrometer to measure the shaker velocity. For example, if we need to obtain a harmonic base acceleration with $1 \mathrm{~g}$ amplitude at $3000 \mathrm{~Hz}$ from the shaker, we send voltages with $3000 \mathrm{~Hz}$ frequency and different amplitudes to the shaker until we find a voltage amplitude that results in $1 g \pm 1 \%$ acceleration. Then we repeat the same procedure for the next frequency step $(3010 \mathrm{~Hz})$. To characterize the shaker for half-sine shock excitations with different time duration, the shaker response is measured with a commercial accelerometer (PCB-352A24). If the shock amplitude is within $\pm 1 \%$ range of the desired amplitude, the voltage will be recorded to be used for the shock experiment. 


\subsection{Harmonic Loading}

After the shaker characterization, we start testing the device by applying harmonic base excitation and performing a frequency sweep. Such an analysis is important to investigate the accelerometer dynamical performance and verify the mathematical model that was developed in Section 3.

The motion of the sensor proof-mass relative to the substrate is obtained by subtracting the substrate response from the device response for each excitation frequency $(f)$. As the motion of the substrate does not depend on the electrical voltage on the device, for each mechanical loading scenario, the substrate motion was measured by reading its velocity with the laser vibrometer. The device response, on the other hand, depends on the mechanical load and electrical voltage on the side electrodes. For each loading case, the proof-mass response was measured again by reading its velocity using the laser vibrometer. Then, the velocity of the substrate for each mechanical load was subtracted from the device response for the corresponding mechanical load to obtain the relative velocity of the proof-mass. Subsequently, the fast Fourier transform (FFT) of this relative velocity signal was calculated to extract the velocity amplitude in the steady-state region. Dividing this velocity amplitude by $2 \pi f$ results in the displacement amplitude for the corresponding frequency. 


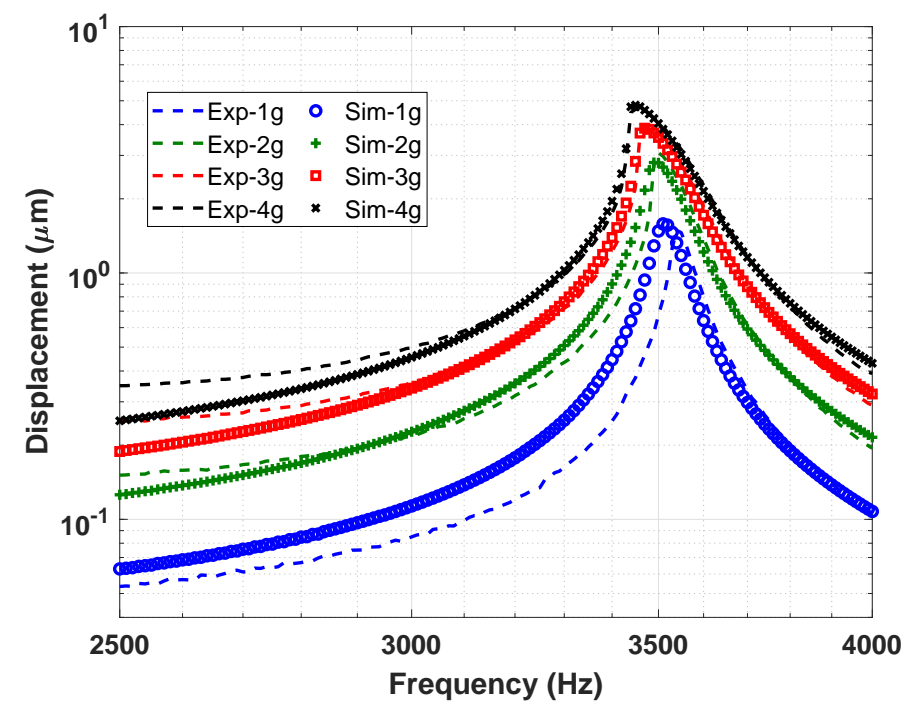

(a) $V d c=60$

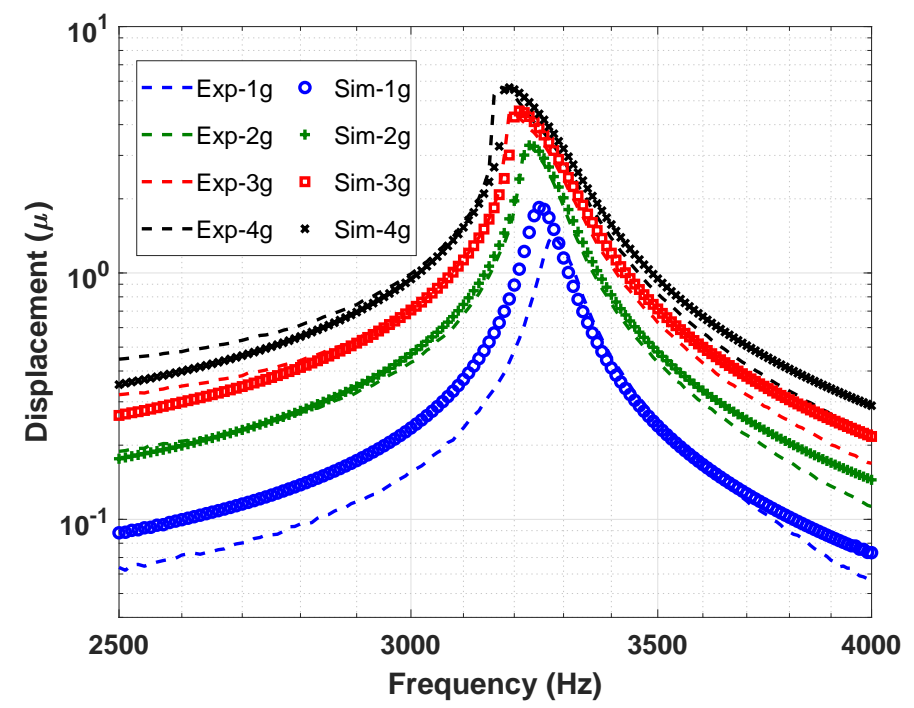

(b) $V d c=50$

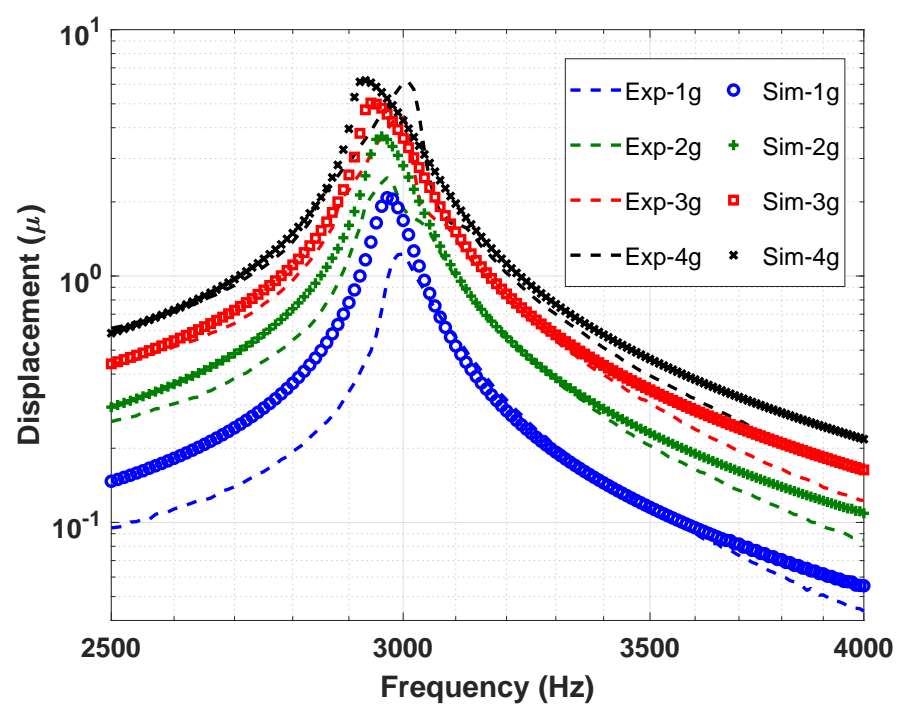

(c) $V d c=40$

Figure 6: Frequency response to harmonic excitation for $V d c=60 \mathrm{~V}, 50 \mathrm{~V}$, and $40 \mathrm{~V}$ and acceleration amplitude of $1-4 g$

Figure 6 shows the device response for different voltages on the side electrodes. For each voltage, different harmonic excitations with different amplitudes are applied. These figures show that the resonance frequency increases as the bias DC voltage increases on the side electrode. Furthermore, for DC voltages of 50 and $60(\mathrm{~V})$, as the amplitude of vibration increases, a nonlinear softening behavior appears in the response. These two phenomena can be explained by looking at nonlinear terms in the electrostatic force. According to Table $1, A_{1}$ (coefficient of $x$ in elec- 
trostatic force) is negative, when it is on the right-hand side of Eq. (1). This means that linear stiffness caused by electrostatic force will add to the linear mechanical stiffness of microstructure, which leads to an increase in stiffness and resonance frequency as DC voltage increases. This property of the microstructure can be beneficial for increased robustness of the sensor against severe mechanical loads. By increasing the DC voltage, the proof-mass plate gets further pushed away from the substrate, hence the maximum mechanical load that is required for the proof-mass to hit the substrate is increased. This means that we can increase the voltage on the side electrodes and use the sensor for more harsh environments in terms of mechanical loads such as mechanical shocks. This is the opposite of what happens to conventional shock sensors that are based on the attractive scheme where an increase in the bias voltage results in early pull-in [20]. Also, one can tune the DC voltage on the side electrodes to obtain the frequency ranges of interest for different applications.

The softening behavior in the frequency response at a high voltage of 50 and $60(V)$ can be explained by the even-order terms in electrostatic force $\left(A_{2}, A_{4}, A_{6}, A_{8}\right)$. Simulation results are presented in Fig. 6. These results are obtained when the linear natural frequency of the structure is set to be the measured value of $1740(\mathrm{~Hz})$ in the governing equation of motion (Eq. (13)). This value is $30 \%$ different from what we obtained from finite element analysis (FEA). This discrepancy can be explained by the imperfections in the fabrication process that are not considered in the FEA. These include a slight bending of the plate that happens because of thermal stresses. The residual stress on the supports plays a significant role on the structural stiffness of the sensor. This residual in-plane stress was measured to be approximately $50 \mathrm{MPa}$ in the fabrication process, which causes a slight curvature in the proof mass plate. The static deflection due to residual stress was measured to be approximately $6 \mu \mathrm{m}$ per $1 \mathrm{~mm}$ length of the plate. Because a static FEA predicted approximately the same deflection as the measured result, we conclude that this deflection is due to in-plane stress only and the variation of residual stress along the thickness may considered to be negligible. Once the estimated linear frequency for the fabricated device is considered in the lumped model, the model captures the experimental results with good accuracy. The slight difference between the simulation results and experimental data, especially at low frequencies, is also attributed to these inevitable fabrication imperfections and residual stresses as well as limitation of approximating a continuous system with a discrete (lumped) model. The damping ratios are adjusted slightly to match the experimental results for each voltage scenario. It is worth mentioning that the squeeze film damping is dependent on the resonance frequency. The resonance frequency changes by varying the DC voltage as shown in Fig.7. Therefore, the damping coefficients will slightly vary with the DC voltage. For the frequency sweep, the lumped model is solved for the dynamic solution $\left(\theta_{d y n}\right)$ using the shooting method [26]. The static rotation caused by DC voltage is calculated and shown in Fig. 8 as a function of DC voltage.

The nonlinearity in the device response when the bias voltage is $40(V)$ is slightly hardening while the lumped model predicts softening. This discrepancy between the simulation results and experimental data can be reconciled by introducing a cubic nonlinear term for mechanical stiffness for this specific voltage but such a nonlinearity makes the response hardening for higher voltages which does not match the experimental data. Also, because the vibration amplitudes with different voltages for the same mechanical loads are very close to each other (for example, the resonance amplitudes for 40 and $50(\mathrm{~V})$ when the acceleration amplitude is $3 g$ ), this difference in 
behavior cannot be attributed to the nonlinearities in the electrostatic force or stiffness caused by air in squeeze film regime. This hardening behavior at low voltages needs further investigation. Although the model does not catch the nonlinear behavior of the microstructure in the resonance region for low voltages, it matches the experimental data away from this region with good accuracy. Because inertial sensors are generally operated in frequency ranges away from their natural frequency the model still could be used to simulate the device response at low voltages.

The sensitivity of the device for different voltages is shown in Fig. 9. The relative displacement of the proof-mass for harmonic base excitation with a different amplitude at $2500(\mathrm{~Hz})$ is recorded and shown in this figure. The slopes of these lines give the mechanical sensitivity for each voltage. The sensitivity for lower voltages is higher because the contribution of electrical stiffness in the total stiffness of the sensor (structural and electrical) is smaller. For example, the sensitivity of the accelerometer at $40 \mathrm{~V}$ is about $0.1 \frac{\mu \mathrm{m}}{\mathrm{g}}$. This is extremely superior to the numbers found in the literature, which are generally in the range of nanometer to femtometer per $\mathrm{g}[27,28]$. In the next step, half-sine shocks will be considered as the mechanical load on the microstructure.

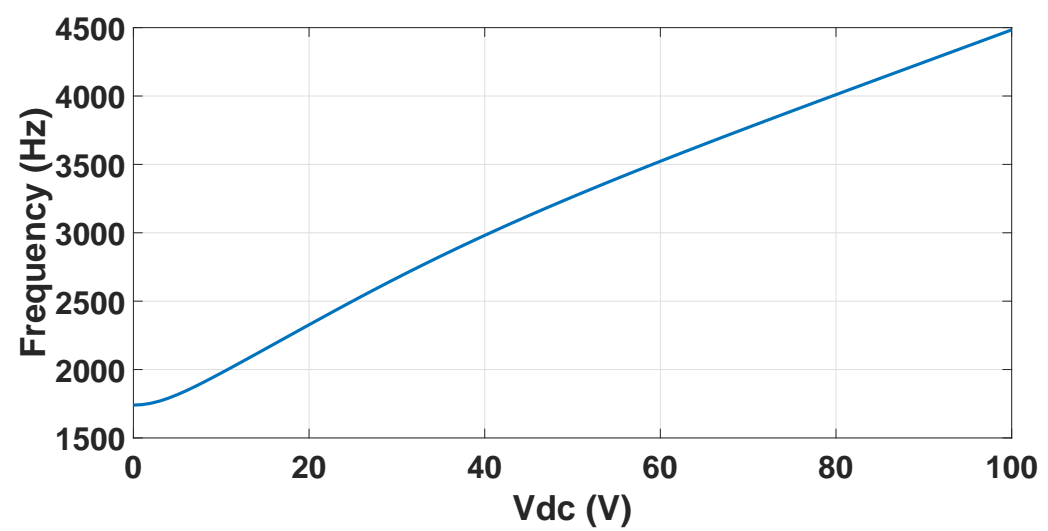

Figure 7: Simulation results for linearized natural frequency for different DC voltages on side electrodes

\subsection{Shock Loading}

Robustness of accelerometers against mechanical shocks is an important parameter that needs thorough investigation. Under mechanical shocks, a force is transmitted to the microstructure through its anchors during a short period of time compared to the natural period of the microstructure. These loads are usually characterized by the induced acceleration on the affected structure with three parameters: maximum acceleration (amplitude), time duration, and pulse shape, also called shock profile. Here, we use half-sine shock loads with different amplitudes and time durations to investigate the response of microstructure to a shock load. The actual output of the shaker is measured with the PCB accelerometer, which is mounted on the shaker. Figure 10 shows this measured acceleration as well as the device response, which is obtained by integrating the velocity of the microstructure measured by the laser vibrometer.

The time responses of the microstructure for $60(\mathrm{~V})$ bias voltage on the side electrode to shock loads with different time durations and amplitudes are given in Fig. 11. Similar experiments were 


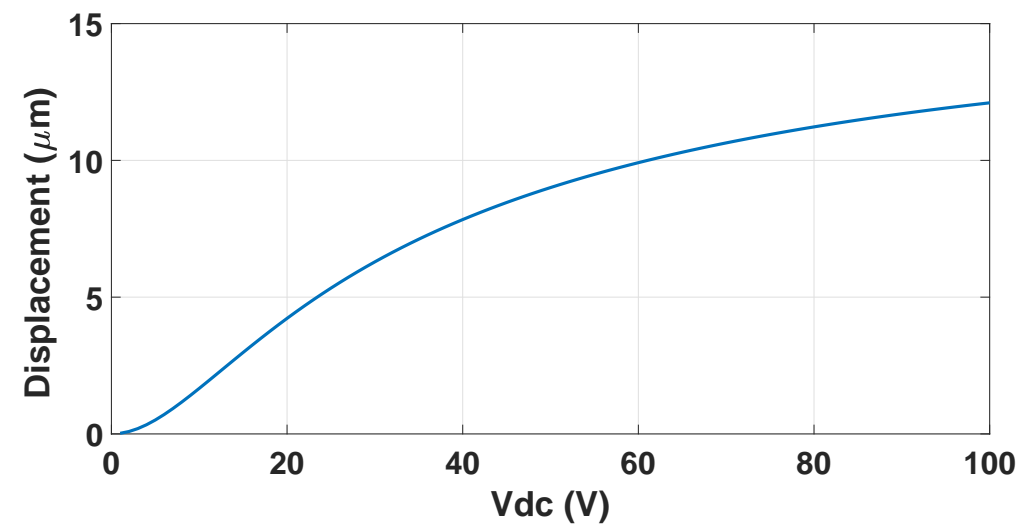

Figure 8: Simulation results for static displacement at the edge of the proof-mass plate for different DC voltages

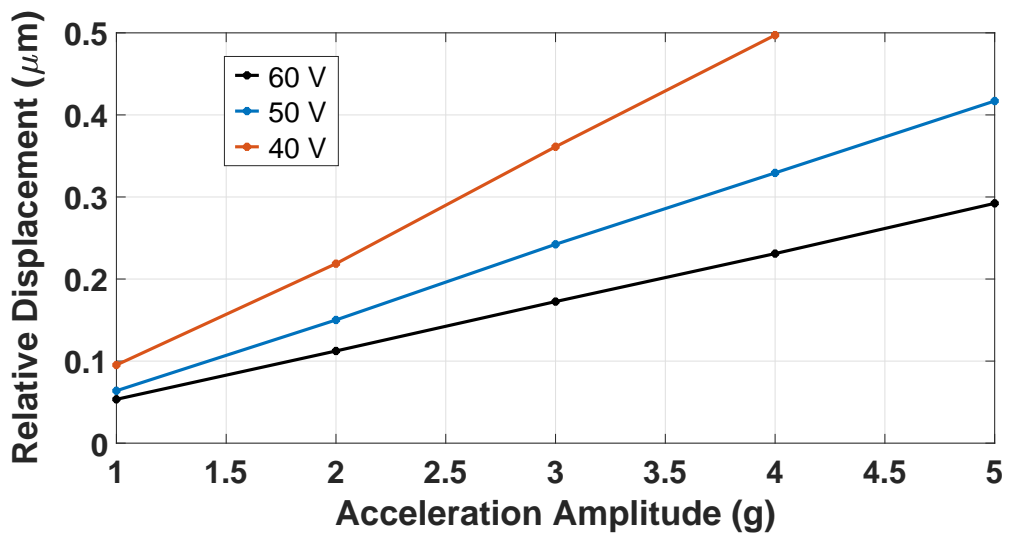

Figure 9: Mechanical sensitivity of the sensor for different DC voltages on the side electrodes obtained from experimental results

conducted for $50(\mathrm{~V})$ on the side electrodes to obtain the larger displacements of the proof-mass (Fig. 12). These shock experiments are done for shock loads that have time durations close to the natural period of the microstructure. The linearized natural frequency of the microstructure for each voltage (Fig. 7) is obtained from stability and eigen-value analysis on the Jacobian matrix for Eq. (13). The linearized natural frequency of the microstructure can be extracted from harmonic experiments at low g levels (Table 5). Because the stiffness of the microstructure and hence, the natural period of the microstructure, would change with the DC voltage, the maximum dynamical displacement of the accelerometer would be a function of the voltage.

The simulation results are in good agreement with the shock-experiment outcomes as shown in Fig. 11 and 12. The small discrepancies can be attributed to the error in the numerical integration of the velocity signal and the fact that the shock that is actually applied on the device is not a perfect half-sine shock considered in the mathematical model. It should also be mentioned that the same damping coefficients that were used for harmonic simulation at 50 and $60(V)$ are used for 


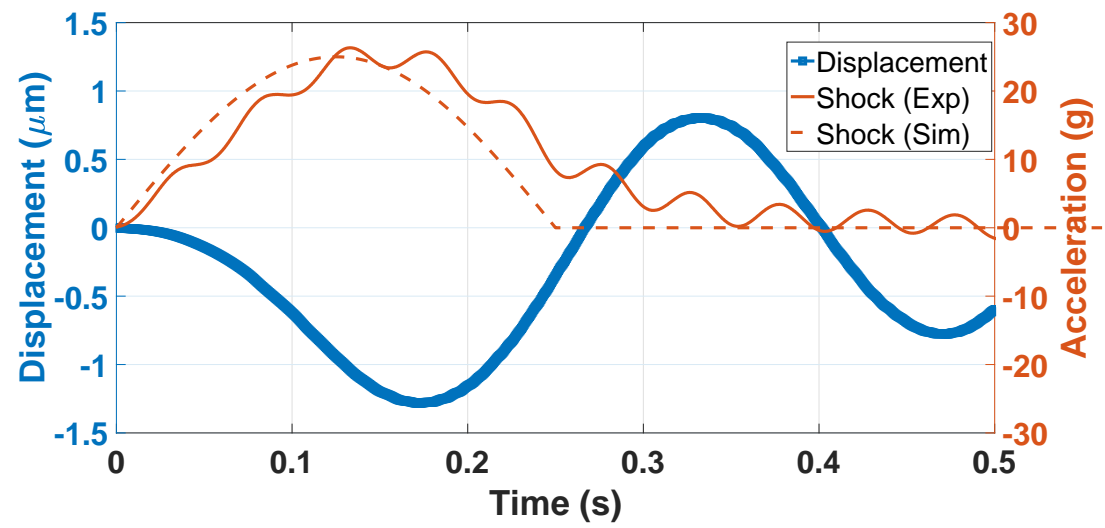

Figure 10: The actual shock in the experiment $(25 g$ and $0.25(\mathrm{~ms}))$, simulated shock profile in the mathematical model and relative displacement at the proof-mass edge for the corresponding shock $\left(V_{d c}=60\right)$

simulating the shock responses. The close agreements of the results show that the damping model can accurately capture the dissipation.

The mechanical sensitivity to shock is an important factor in the performance of shock sensors. According to Figure 11 at $60(\mathrm{~V})$, the maximum displacement at the proof-mass edge is less than $1.2 \mu \mathrm{m}$ when the shock amplitude is $25 \mathrm{~g}$. If we consider the collision of the microstructure with its substrate as a failure mode or a limit to define the maximum allowable shock load, this $1.2 \mu \mathrm{m}$ is $10 \%$ of the initial gap between the proof-mass edge and the substrate ( $2 \mu \mathrm{m}$ fabrication gap + $10 \mu \mathrm{m}$ static gap caused by $60(\mathrm{~V})$ applied to the side electrodes). However, as discussed in sections 1 and 2, because there is no pull-in with this repulsive approach, even if the proof-mass hits the substrate, it could bounce off safely. In fact, if this impact could be detected electrically, the device could be employed as a tunable threshold shock sensor. As opposed to conventional parallel-plate shock sensors, the threshold shock could be tuned by applying voltage on the side electrodes. This capability is enabled in the repulsive configuration because of the additional two electrodes on the sides.

Because of the limitation on the shock amplitudes that could be generated by the shaker, we could not test the device under more severe shocks. However, we can use the developed model to predict the threshold shock at different voltages. These threshold shock amplitudes are given in Table 5 for different voltages on side electrodes. As discussed in Section 5.2, the sensor resilience against mechanical shock could be improved by increasing the voltage on the side electrodes. This also means the threshold shock the sensor can detect can be tuned by varying the DC voltage on the side electrodes.

\section{Conclusion}

In this study, a capacitive electrode system that achieves a repulsive electrostatic force is employed for acceleration-sensing for the first time. An accelerometer is simulated and fabricated. A single degree of freedom lumped parameter model is constructed that captures the nonlinear dy- 


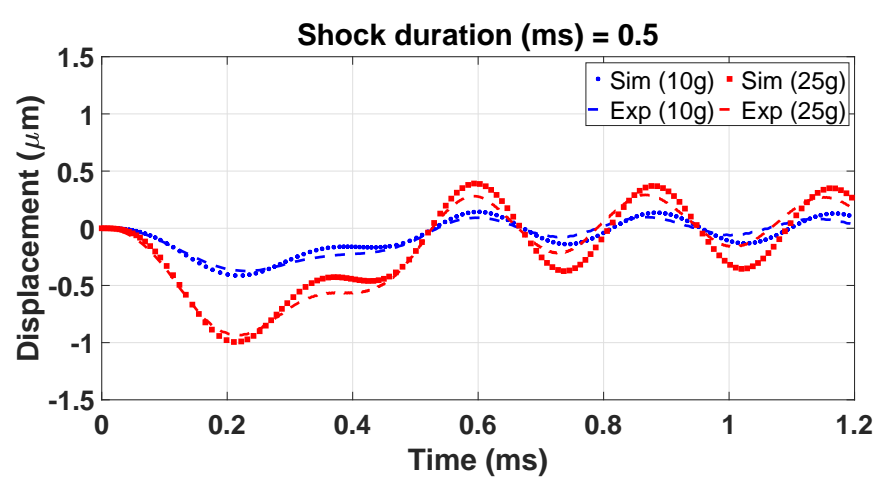

(a) $\frac{T_{S h}}{T_{n}}=1.79$

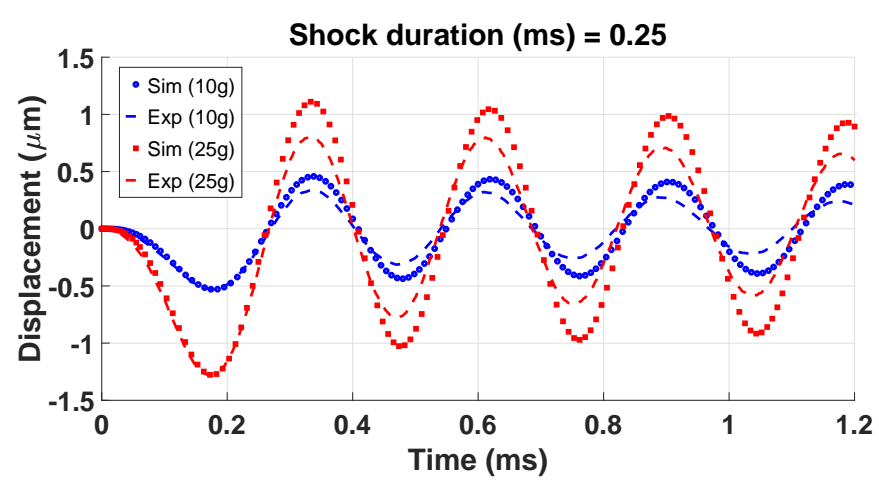

(c) $\frac{T_{S h}}{T_{n}}=0.89$

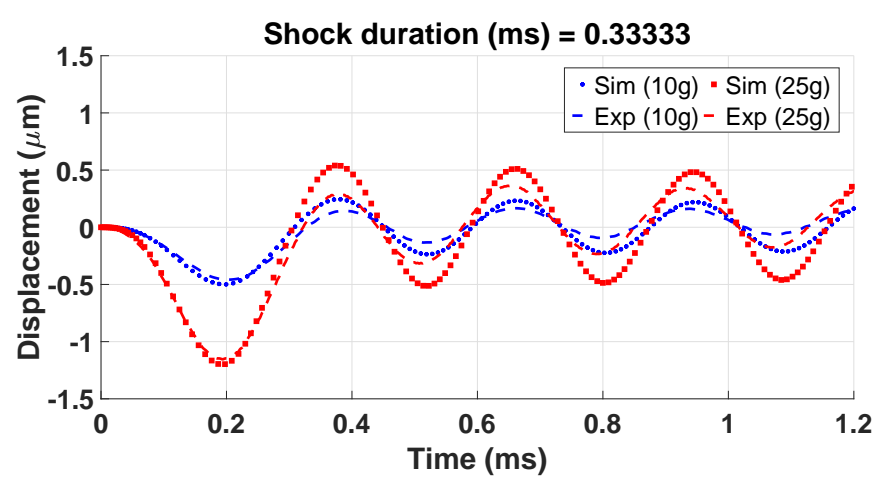

(b) $\frac{T_{S h}}{T_{n}}=1.18$

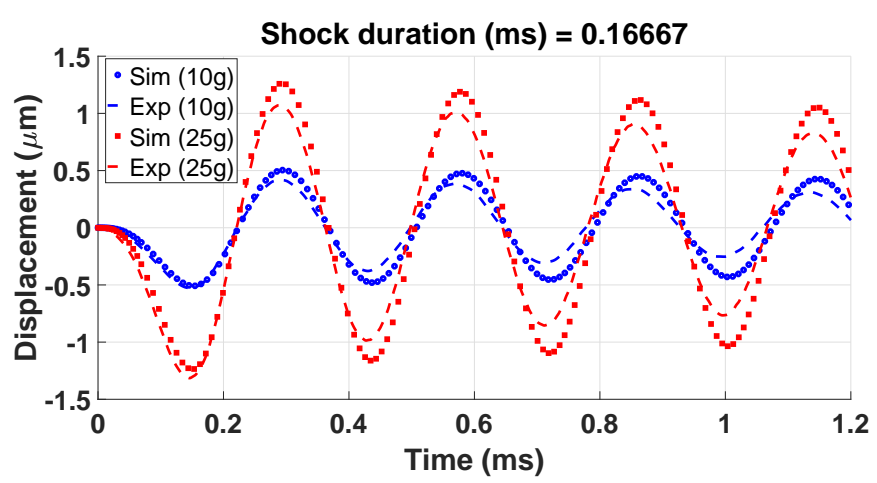

(d) $\frac{T_{S h}}{T_{n}}=0.61$

Figure 11: Simulation and experimental time responses for half sine shocks with different time duration at $60(\mathrm{~V})$ bias voltages. (a) $\frac{T_{S h}}{T_{n}}=1.79$, (b) $\frac{T_{S h}}{T_{n}}=1.18$, (c) $\frac{T_{S h}}{T_{n}}=0.89$, (d) $\frac{T_{S h}}{T_{n}}=0.61$.

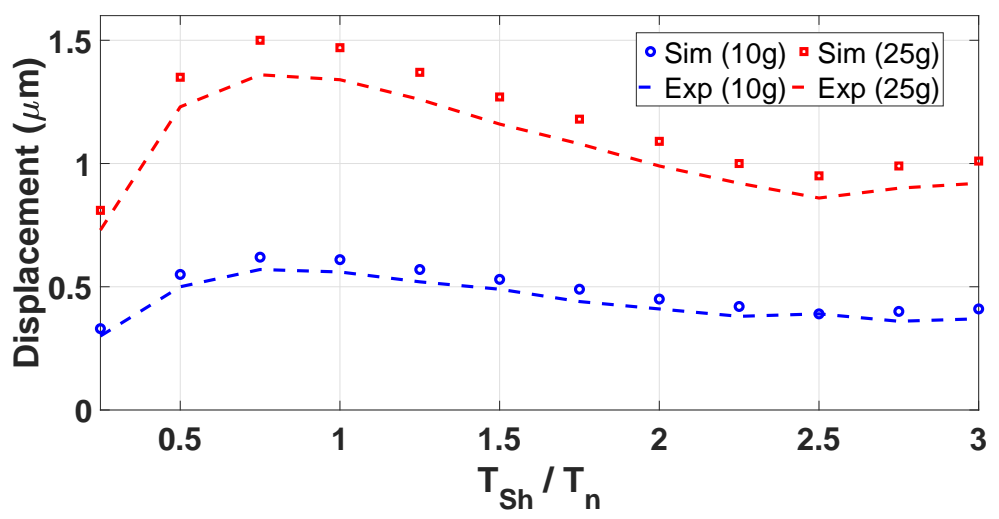

Figure 12: Simulation and experimental time responses for half sine shocks with time duration $\left(T_{s h}\right)$ equal to $25 \%-300 \%$ of the natural period of the microstructure $\left(T_{n}\right)$ at $50(\mathrm{~V})$ bias voltages

namics of the system. Comprehensive dynamic analysis of the microstructure under electrostatic force and different mechanical loads is investigated. As the presented accelerometer does not suf- 


\begin{tabular}{|c|c|c|c|c|}
\hline$V_{d c}(V)$ & $\begin{array}{c}\text { Threshold } \\
\text { shock (g) }\end{array}$ & $\begin{array}{c}\text { Resonance } \\
\text { requency (Hz) } \\
\text { (experiment) }\end{array}$ & $\begin{array}{c}\text { Resonance } \\
\text { frequency (Hz) } \\
\text { (simulation) }\end{array}$ & Error (\%) \\
\hline 40 & 200 & 3000 & 2981 & 0.64 \\
50 & 300 & 3280 & 3262 & 0.55 \\
60 & 410 & 3540 & 3523 & 0.67 \\
\hline
\end{tabular}

Table 5: Threshold shock amplitude that results in proof-mass hitting the substrate (Shock duration $=90 \%$ of natural period of microstructure for each voltage) and comparing resonance frequencies obtained from experiments (at low $\mathrm{g}$ $(1 \mathrm{~g})$ ) with linearized natural frequencies derived from Jacobian matrix

fer from pull-in, the DC voltage can be increased to increase the fundamental natural frequency. This capability enables the accelerometer to become tunable. That means the detection range of the accelerometers, which is often below one third of their natural frequency, can be tuned by changing the DC voltage of the side electrodes. This is superior to current commercial accelerometers that often have a fixed resonance frequency, which limits their performance. Here, because the resonance frequency of the accelerometer can be tuned, it has the potential to be used in a wider range of applications. Furthermore, the initial gap between the proof-mass and the substrate is increased by increasing the voltage on the side electrodes, which improves the accelerometer robustness against mechanical shocks without sacrificing its stability. This device also has the potential to be designed and used as a shock sensor. By changing the voltage difference between the side and bottom electrodes, the threshold shock amplitude needed to collide the structure with the substrate can be tuned. Also, the natural period of the sensor can be tuned to use the sensor for shock loads with different time duration. In the harmonic and shock cases, simulations are validated by experimental results.

\section{Acknowledgment}

The authors would like to greatly acknowledge the National Science Foundation (NSF) for its support through ECCS grant \#1608692. Authors are thankful to the Cornell NanoScale Science $\&$ Technology Facility (CNF) for use of their micro-fabrication facility and their help during the fabrication process. Also, the authors would like to thank Dr. Weili Cui and Dr. Mark Pallay for their fruitful discussions.

\section{References}

[1] D. W. Burns, J. D. Zook, R. D. Horning, W. R. Herb, H. Guckel, Sealed-cavity resonant microbeam pressure sensor, Sensors and actuators A: Physical 48 (3) (1995) 170-186.

[2] O. J. Woodman, An introduction to inertial navigation, University of Cambridge, 2007 (696).

[3] W. D. Cowan, M. K. Lee, B. M. Welsh, V. M. Bright, M. C. Roggemann, Surface micromachined segmented mirrors for adaptive optics, IEEE Journal of Selected Topics in quantum Electronics 5 (1) (1999) 90-101.

[4] M. I. Younis, MEMS Linear and Nonlinear Statics and Dynamics, Vol. 20, New York, USA, Springer, 2011.

[5] G. N. Nielson, G. Barbastathis, Dynamic pull-in of parallel-plate and torsional electrostatic mems actuators, Journal of Microelectromechanical Systems 15 (4) (2006) 811-821.

[6] R. N. Miles, W. Cui, Q. T. Su, D. Homentcovschi, A MEMS low-noise sound pressure gradient microphone with capacitive sensing, Journal of Microelectromechanical Systems 24 (2015) 241-248. 
[7] S. Towfighian, A. Seleim, E. Abdel-Rahman, G. Heppler, A large-stroke electrostatic micro-actuator, Journal of Micromechanics and Microengineering 21 (7) (2011) 075023.

[8] S. He, R. Ben Mrad, Large-stroke microelectrostatic actuators for vertical translation of micromirrors used in adaptive optics, IEEE Transactions on Industrial Electronics 52 (4) (2005) 974-983.

[9] S. He, R. Ben Mrad, Design, modeling, and demonstration of a MEMS repulsive-force out-of-plane electrostatic micro actuator, Journal of Microelectromechanical Systems 17 (3) (2008) 532-547.

[10] J. Zhou, R. N. Miles, S. Towfighian, A novel capacitive sensing principle for microdevices, in: ASME 2015 International Design Engineering Technical Conferences and Computers and Information in Engineering Conference, American Society of Mechanical Engineers.

[11] S. Sundaram, M. Tormen, B. Timotijevic, R. Lockhart, T. Overstolz, R. P. Stanley, H. R. Shea, Vibration and shock reliability of MEMS: modeling and experimental validation, Journal of Micromechanics and Microengineerin 21 (2011) 045022.

[12] K. B. Lee, Y.-H. Cho, Laterally driven electrostatic repulsive-force microactuators using asymmetric field distribution, Journal of Microelectromechanical Systems 10 (1) (2001) 128-136.

[13] S. He, R. Ben Mrad, Development of a bi-directional micro electrostatic comb actuator for optical mems devices, Proceedings of the SPIE Opto-Canada Conference, Ottawa, Canada, May 9-10, 2002.

[14] S. He, R. Ben Mrad, Development of a novel translation micromirror for adaptive optics, Proceedings of SPIE The International Society for Optical Engineering, October, 2003.

[15] M. Pallay, M. Daeichin, S. Towfighian, Dynamic behavior of an electrostatic MEMS resonator with repulsive actuation, Nonlinear Dynamics 89 (2) (2017) 15251538.

[16] M. Ozdogan, M. Daeichin, A. Ramini, S. Towfighian, Parametric resonance of a repulsive force MEMS electrostatic mirror, Sensors and Actuators A: Physical 265 (2017) 20-31.

[17] M. Pallay, S. Towfighian, A parametric electrostatic resonator using repulsive force, Sensors and Actuators A: Physical 277 (2018) 134-141.

[18] D. S. Eddy, dougLas R. Sparks, Application of MEMS technology in automotive sensors and actuators, Proceedings of the IEEE 86 (8) (1998) 1747-1755.

[19] A. Ibrahim, M. I. Younis, Simple fall criteria for MEMS sensors: Data analysis and sensor concept, Sensors (2014) 12149-73.

[20] M. I. Younis, F. M. Alsaleem, R. Miles, Q. Su, Characterization for the performance of capacitive switches activated by mechanical shock, Journal of Micromechanics and Microengineering (2011) 1360-1370.

[21] J. B. Starr, Squeeze-film damping in solid-state accelerometers, Proceeding of the IEEE Solid-State Sensor and Actuator Workshop.

[22] J. J. Blech, On isothermal squeeze films, Journal of Lubrication Technology (1983) 615-620.

[23] W. S. Griffin, H. H. Richardson, S. Yamanami, A study of fluid squeeze-film damping, Journal of Basic Engineering (1966) 451-456.

[24] P. French, Polysilicon: a versatile material for microsystems, Sensors and actuators A: Physical 99 (1) (2002) 3-12.

[25] W. Cui, Analysis, design and fabrication of a novel silicon microphone, PhD Dissertation, SUNY Binghamton, 2004.

[26] A. H. Nayfeh, B. Balachandran, Applied Nonlinear Dynamics, Virginia, USA, Wiley, 2007.

[27] R. H. Olsson, K. E. Wojciechowski, M. S. Baker, M. R. Tuck, J. G. Fleming, Post-cmos-compatible aluminum nitride resonant mems accelerometers, Journal of Microelectromechanical Systems 18 (3) 2009.

[28] H. Luo, G. Zhang, L. R. Carley, G. K. Fedder, A post-cmos micromachined lateral accelerometer, Journal of Microelectromechanical Systems 11 (3) (2002) 188-195. 